\title{
Two-Photon Decay of the Neutral Pion in Lattice QCD
}

\author{
Xu Feng, ${ }^{1}$ Sinya Aoki, ${ }^{2}$ Hidenori Fukaya, ${ }^{3}$ Shoji Hashimoto, ${ }^{1,4}$ Takashi Kaneko, ${ }^{1,4}$ \\ Jun-ichi Noaki, ${ }^{1}$ and Eigo Shintani ${ }^{5}$
}

(JLQCD Collaboration)

\author{
${ }^{1}$ High Energy Accelerator Research Organization (KEK), Tsukuba 305-0801, Japan \\ ${ }^{2}$ Graduate School of Pure and Applied Sciences, University of Tsukuba, Tsukuba 305-8571, Japan \\ ${ }^{3}$ Department of Physics, Osaka University, Toyonaka, Osaka 560-0043, Japan \\ ${ }^{4}$ School of High Energy Accelerator Science, The Graduate University for Advanced Studies (Sokendai), \\ Tsukuba 305-0801, Japan \\ ${ }^{5}$ RIKEN-BNL Research Center, Brookhaven National Laboratory, Upton, New York 11973, USA
}

(Received 7 June 2012; published 2 November 2012)

\begin{abstract}
We perform a nonperturbative calculation of the $\pi^{0} \rightarrow \gamma \gamma$ transition form factor and the associated decay width using lattice QCD. The amplitude for a two-photon final state, which is not an eigenstate of QCD, is extracted through a Euclidean time integral of the relevant three-point function. We utilize the allto-all quark propagator technique to carry out this integration as well as to include the disconnected quark diagram contributions. The overlap fermion formulation is employed on the lattice to ensure exact chiral symmetry on the lattice. After examining various sources of systematic effects, except for a possible discretization effect, we obtain $\Gamma_{\pi^{0} \rightarrow \gamma \gamma}=7.83(31)(49) \mathrm{eV}$ for the pion decay width, where the first error is statistical and the second is our estimate of the systematic error.
\end{abstract}

DOI: 10.1103/PhysRevLett.109.182001

PACS numbers: $12.38 . \mathrm{Gc}, 12.40 . \mathrm{Vv}, 13.25 . \mathrm{Cq}$

The neutral pion decay process provides a unique opportunity to test a fundamental symmetry property of the gauge theory. A quantum effect due to a fermion loop violates the axial-current conservation, and gives the dominant contribution to the $\pi^{0} \rightarrow \gamma \gamma$ decay rate. The prediction from this Adler-Bell-Jackiw (ABJ) anomaly $[1,2]$ (or the chiral anomaly) is rather precise because higher-loop diagrams do not contribute in the limit of vanishing quark mass and external momenta [3] even under the presence of strong interaction. On the other hand, a recent experimental measurement of the neutral pion decay width has reached the accuracy of 2.8\% [4] and is aiming to achieve $1.4 \%$ in the near future. At this level of accuracy, the correction due to finite quark mass becomes relevant. Phenomenologically, an enhancement of the decay width of about $3-5 \%$ has been expected according to the sum rule and chiral perturbation theory $(\chi \mathrm{PT})$ approaches [5-8], in which the main effect comes from a mixing of $\pi^{0}$ with $\eta$ and $\eta^{\prime}$ mesons. For a recent review, we refer the reader to Ref. [9].

In this Letter we present a model-independent calculation of the $\pi^{0} \rightarrow \gamma \gamma$ amplitude using the lattice quantum chromodynamics including dynamical up, down, and strange quarks. We use the overlap fermion formulation [10], which preserves chiral symmetry at finite lattice spacings. In this formulation, the chiral anomaly appears through the Jacobian of chiral transformation; the AtiyahSinger index theorem is reproduced as far as the background gauge field is smooth enough [11]. On the other hand, whether the chiral anomaly is correctly reproduced at practically used lattice spacings $(\sim 0.1 \mathrm{fm})$ is a nonperturbative problem, one that we address in this work.

Compared to previous attempts [12,13], a new technique is applied to treat the two-external-photon state, by utilizing the all-to-all quark propagator $[14,15]$. In the limit of degenerate up and down quark masses, we obtain the decay rate with a statistical error of $4 \%$ and a total error of $7 \%$ after examining possible systematic effects.

The $\pi^{0} \rightarrow \gamma \gamma$ decay rate at the leading order of QED can be expressed as

$$
\Gamma_{\pi^{0} \gamma \gamma}=\frac{\pi \alpha_{e}^{2} m_{\pi}^{3}}{4} \mathcal{F}_{\pi^{0} \gamma \gamma}^{2}\left(m_{\pi}^{2}, 0,0\right)
$$

where $\alpha_{e}$ is the fine structure constant, $m_{\pi}$ is the neutral pion mass, and $\mathcal{F}_{\pi^{0} \gamma \gamma}\left(m_{\pi}^{2}, p_{1}^{2}, p_{2}^{2}\right)$ is the form factor of the pion to two (virtual) photon transition with $p_{1,2}$ the photon momenta. In the chiral limit, the ABJ anomaly predicts

$$
\mathcal{F}_{\pi^{0} \gamma \gamma}^{\mathrm{ABJ}} \equiv \mathcal{F}_{\pi^{0} \gamma \gamma}(0,0,0)=\frac{1}{4 \pi^{2} F_{0}},
$$

where $F_{0}$ is the pion decay constant $F_{\pi}$ in the chiral limit. We define a normalized form factor as $F\left(m_{\pi}^{2}, p_{1}^{2}, p_{2}^{2}\right) \equiv$ $\left(4 \pi^{2} F_{\pi}\right) \mathcal{F}_{\pi^{0} \gamma \gamma}\left(m_{\pi}^{2}, p_{1}^{2}, p_{2}^{2}\right)$. In the Minkowski space-time, $\mathcal{F}_{\pi^{0} \gamma \gamma}\left(m_{\pi}^{2}, p_{1}^{2}, p_{2}^{2}\right)$ is defined through the matrix element 


$$
\begin{aligned}
M_{\mu \nu}\left(p_{1}, p_{2}\right) & =i \int d^{4} x e^{i p_{1} x}\left\langle\Omega\left|T\left\{j_{\mu}(x) j_{\nu}(0)\right\}\right| \pi^{0}(q)\right\rangle \\
& =\varepsilon_{\mu \nu \alpha \beta} p_{1}^{\alpha} p_{2}^{\beta} \mathcal{F}_{\pi^{0} \gamma \gamma}\left(m_{\pi}^{2}, p_{1}^{2}, p_{2}^{2}\right),
\end{aligned}
$$

where $q$ is the $\pi^{0}$ momentum satisfying the on-shell condition $q^{2}=m_{\pi}^{2}$. The current $j_{\mu}=\sum_{f} Q_{f} \bar{q}_{f} \gamma_{\mu} q_{f}$ is the hadronic component of the electromagnetic vector current and the sum runs over all relevant quark flavors: $f=u, d, s . Q_{f}$ denotes the electromagnetic charge of them: $Q_{u}=+2 / 3$ and $Q_{d, s}=-1 / 3$. The factor $\varepsilon_{\mu \nu \alpha \beta} p_{1}^{\alpha} p_{2}^{\beta}$ is induced by the negative parity of $\pi^{0}$.

By an analytic continuation of (3) from the Minkowski to Euclidean space-time [16,17], one may write

$$
\begin{aligned}
M_{\mu \nu}\left(p_{1}, p_{2}\right)= & \lim _{t_{1,2}-t_{\pi} \rightarrow \infty} \frac{1}{\frac{\phi_{\pi, \vec{q}}}{2 E_{\pi, \vec{q}}} e^{-E_{\pi, \vec{q}}\left(t_{2}-t_{\pi}\right)}} \\
& \times \int d t_{1} e^{\omega\left(t_{1}-t_{2}\right)} C_{\mu \nu}\left(t_{1}, t_{2}, t_{\pi}\right), \\
C_{\mu \nu}\left(t_{1}, t_{2}, t_{\pi}\right) \equiv & \int d^{3} \vec{x} e^{-i \vec{p}_{1} \cdot \vec{x}} \int d^{3} \vec{z} e^{i \vec{q} \cdot \vec{z}} \\
& \times\left\langle\Omega\left|T\left\{j_{\mu}\left(\vec{x}, t_{1}\right) j_{\nu}\left(\overrightarrow{0}, t_{2}\right) \pi^{0}\left(\vec{z}, t_{\pi}\right)\right\}\right| \Omega\right\rangle,
\end{aligned}
$$

where $t_{1}, t_{2}$, and $t_{\pi}$ are Euclidean time slices. $\int d^{3} \vec{z} e^{i \vec{q} \cdot \vec{z}} \pi^{0}\left(\vec{z}, t_{\pi}\right)$ is an interpolating operator for the neutral pion with the spatial momentum $\vec{q}$. Its amplitude and energy in the ground state are denoted by $\phi_{\pi, \vec{q}}$ and $E_{\pi, \vec{q}}$, respectively. The four-momentum of the first photon $p_{1}=\left(\omega, \vec{p}_{1}\right)$ is chosen as input, while the momentum of the second photon is given as $p_{2}=\left(E_{\pi, \vec{q}}-\omega, \vec{q}-\vec{p}_{1}\right)$ by momentum conservation. Note that the analytical continuation is valid only for $p_{1,2}^{2}<M_{V}^{2}$, which sets the limit on the value of $\omega .\left(M_{V}\right.$ stands for the invariant mass of the lowest energy state in the vector channel.) Since the two photons cannot be on-shell simultaneously, we calculate the form factor at the off-shell photon momenta and then extrapolate to the on-shell limit.

To calculate the matrix element $\left\langle\Omega\left|j_{\mu} j_{\nu} \pi^{0}\right| \Omega\right\rangle$ in Eq. (4), we use $2+1$-flavor overlap fermion configurations generated by the JLQCD and TWQCD Collaborations $[18,19]$ at a single lattice spacing $a=0.11 \mathrm{fm}$ and two spatial lattice sizes $L / a=16$ and 24 . The time extent is $T / a=48$. Although the main gauge ensembles have a fixed (global) topological charge $Q=0$, the deviation from the $\theta$ vacuum is understood as a finite volume effect of $O\left(1 / L^{3} T\right)$ [20]. We check the significance of this effect by comparing the results with two different values $Q=0,1$. We utilize the all-to-all propagator to calculate the correlation function $C_{\mu \nu}\left(t_{1}, t_{2}, t_{\pi}\right)$ at any time slices of $t_{1}, t_{2}$, and $t_{\pi}$. The electromagnetic current $j_{\mu}=\sum_{f} Q_{f} \bar{q}_{f} \gamma_{\mu} q_{f}$ is implemented on the lattice as a local operator with a renormalization factor calculated nonperturbatively in [21] to match the lattice results with the continuum theory. The up and down quarks are degenerate in mass. We use the bare values $a m_{u, d}=0.015,0.025,0.035$, and 0.050 , corresponding to the pion mass $m_{\pi}$ ranging from 290 to $540 \mathrm{MeV}$. Our final results are obtained by an extrapolation of the data to the physical pion mass $m_{\pi \text {,phy }}$. The strange quark mass is fixed at $a m_{s}=0.080$, which is very close to the estimated physical value.

From the large $t_{1,2}-t_{\pi}$ behavior of $C_{\mu \nu}\left(t_{1}, t_{2}, t_{\pi}\right)$, it is possible to extract the $\pi^{0}$ ground state. We define the amplitude $A_{\pi}$ as

$$
A_{\pi}(\tau) \equiv \lim _{t-t_{\pi} \rightarrow \infty} C_{\mu \nu}\left(t_{1}, t_{2}, t_{\pi}\right) / e^{-E_{\pi, \vec{q}}\left(t-t_{\pi}\right)},
$$

with $\tau=t_{1}-t_{2} \quad$ and $\quad t=\min \left\{t_{1}, t_{2}\right\}, \quad$ and obtain $M_{\mu \nu}\left(p_{1}, p_{2}\right)$ by performing an intregation,

$$
\frac{2 E_{\pi, \vec{q}}}{\phi_{\pi}}\left(\int_{0}^{\infty} d \tau e^{\omega \tau} A_{\pi}(\tau)+\int_{-\infty}^{0} d \tau e^{\left(\omega-E_{\pi, \vec{q}}\right) \tau} A_{\pi}(\tau)\right) .
$$

We use two momentum setups $\vec{p}_{1}=\frac{2 \pi}{L}(0,0,0), \vec{q}=$ $\frac{2 \pi}{L}(0,0,1)$ (setup 1) and $\vec{p}_{1}=\frac{2 \pi}{L}(0,0,1), \vec{q}=\frac{2 \pi}{L}(0,0,0)$ (setup 2). The resulting amplitudes $A_{\pi}(\tau)$ for these setups are shown in Fig. 1. In order to qualitatively understand the $\tau$ dependence of the $A_{\pi}(\tau)$, we consider the vectormeson-dominance (VMD) model $\mathcal{F}_{\pi^{0} \gamma \gamma}^{\mathrm{VMD}}\left(m_{\pi}^{2}, p_{1}^{2}, p_{2}^{2}\right)=$ $c_{V} G_{V}\left(p_{1}^{2}\right) G_{V}\left(p_{2}^{2}\right)$, with $G_{V}\left(p^{2}\right)=M_{V}^{2} /\left(M_{V}^{2}-p^{2}\right)$ the vector-meson propagator and $c_{V}$ a constant. The amplitude $A_{\pi}^{\mathrm{VMD}}(\tau)$, reconstructed from this model, is plotted by red solid curves in Fig. 1. (The detailed expression for $A_{\pi}^{\mathrm{VMD}}(\tau)$ will be given in a later publication [22]). We find that the VMD model describes the lattice data at $|\tau| / a=7$, and we can safely evaluate the contribution beyond $|\tau| / a=13$, where the lattice data are truncated due to the finite time extent $T$. At small $|\tau|$, the VMD model fails to match the lattice data. This is because no information about the vector-meson excited states is contained in $\mathcal{F}_{\pi^{0} \gamma \gamma}^{\mathrm{VMD}}\left(m_{\pi}^{2}, p_{1}^{2}, p_{2}^{2}\right)$. Given the dominant role played by
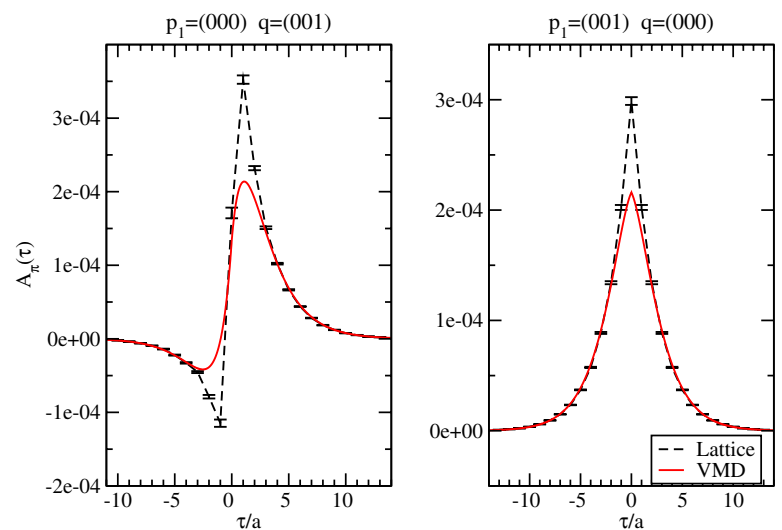

FIG. 1 (color online). The amplitude $A_{\pi}(\tau)$ as a function of $\tau$ for momentum setup 1 (left) and setup 2 (right). The black dashed (red solid) curves indicate the lattice (VMD) amplitudes. 

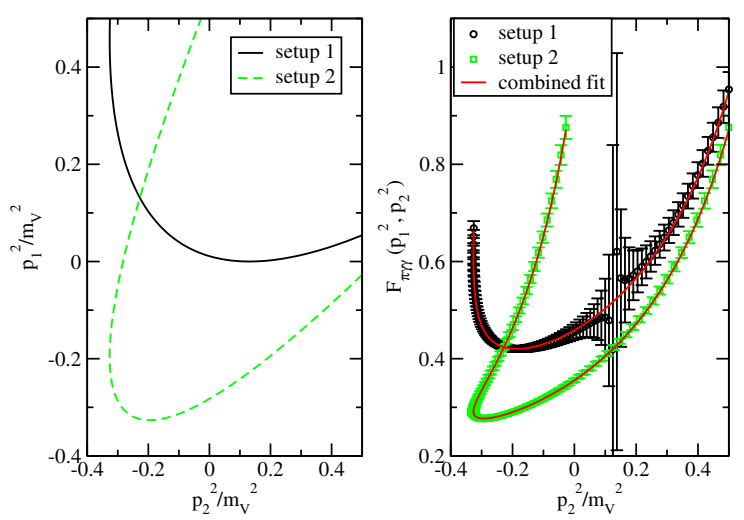

FIG. 2 (color online). Left: contour of $\left(p_{1}^{2}, p_{2}^{2}\right)$ rescaled by $1 / M_{V}^{2}$ for our momentum setups. Right: $F_{\pi^{0} \gamma \gamma}\left(m_{\pi}^{2}, p_{1}^{2}, p_{2}^{2}\right)$ as a function of $p_{2}^{2} / M_{V}^{2}$. Lattice data are obtained on a $24^{3} \times 48$ lattice at $a m_{u, d}=0.015$.

the lowest vector meson, we take its form as a basis to analyze the functional form of $\mathcal{F}_{\pi^{0} \gamma \gamma}\left(m_{\pi}^{2}, p_{1}^{2}, p_{2}^{2}\right)$. Namely, we use an expression

$$
\begin{aligned}
\mathcal{F}_{\pi^{0} \gamma \gamma}\left(m_{\pi}^{2}, p_{1}^{2}, p_{2}^{2}\right) & \\
= & c_{V} G_{V}\left(p_{1}^{2}\right) G_{V}\left(p_{2}^{2}\right)+\sum_{m} c_{m}\left[\left(p_{2}^{2}\right)^{m} G_{V}\left(p_{1}^{2}\right)\right. \\
& \left.+\left(p_{1}^{2}\right)^{m} G_{V}\left(p_{2}^{2}\right)\right]+\sum_{m, n} c_{m, n}\left(p_{1}^{2}\right)^{m}\left(p_{2}^{2}\right)^{n}
\end{aligned}
$$

which includes possible contributions from excited states as a polynomial of $p_{1,2}^{2}$. In the chiral and large volume limit for which the two-pion threshold opens, the VMD model would no longer give an adequate description of the data. Such an effect could become significant for precision better than a few percent, which is not within the scope of this work.

By varying $\omega$, we obtain $M_{\mu \nu}\left(p_{1}, p_{2}\right)$ in a certain range of $p_{1}^{2}$ and $p_{2}^{2}$. As shown in the left panel of Fig. 2, a pair $\left(p_{1}^{2}, p_{2}^{2}\right)=\left[\omega^{2}-\vec{p}_{1}^{2},\left(E_{\pi, \vec{q}}-\omega\right)^{2}-\left(\vec{q}-\vec{p}_{1}\right)^{2}\right]$ forms a continuous contour on the $\left(p_{1}^{2}, p_{2}^{2}\right)$ plane for $p_{1,2}^{2}<$ $M_{V}^{2} / 2$. Evaluating $\mathcal{F}_{\pi^{0} \gamma \gamma}\left(m_{\pi}^{2}, p_{1}^{2}, p_{2}^{2}\right)$ along this contour, we obtain the data plotted in the right panel of Fig. 2. We perform the combined fit of these data to Eq. (7) with four free parameters: $c_{V}, c_{0}, c_{0,0}$, and $c_{0,1}=c_{1,0}$, truncating the higher-order terms which turned out to be negligibly small. The fitting curves are shown in the right panel of Fig. 2. As expected, the single formula (7) describes the data with different momentum setups. Combining the resulting fit parameters, we obtain the normalized form factors $F\left(m_{\pi}^{2}, 0,0\right)$, which are plotted in the uppermost panel of Fig. 3.

In the following, we analyze the details of systematic effects. When calculating the integral in Eq. (6), we use the summation instead of the integration. This causes a discretization effect, which vanishes in the continuum limit. Putting $A_{\pi}^{\mathrm{VMD}}(\tau)$ into Eq. (6), we find that the fractional

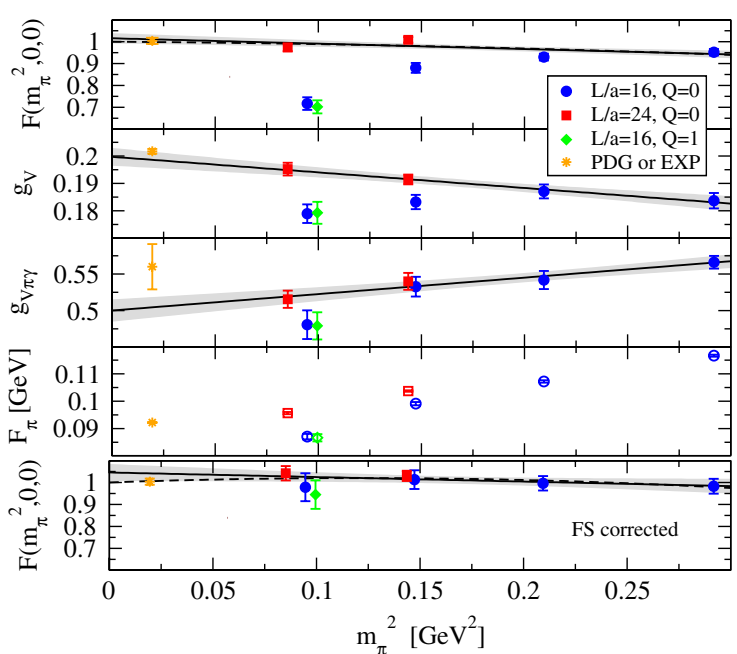

FIG. 3 (color online). $F\left(m_{\pi}^{2}, 0,0\right), g_{V}, g_{V \pi \gamma}, F_{\pi}$, and FS corrected $F\left(m_{\pi}^{2}, 0,0\right)$ as a function of $m_{\pi}^{2}$ from top to bottom panels. In each panel, data with $(L / a, Q)=(16,0),(24,0)$, and $(16,1)$ are plotted by the blue circles, red squares, and green diamonds, respectively. The yellow stars indicate the Particle Data Group (PDG) [27] or PrimEx Collaboration [4] experimental values, for reference. The solid (dashed) curves show the result of the fit to the linear (quadratic) function. The data set used in the fit is explained in the text.

difference between $M_{\mu \nu}\left(p_{1}, p_{2}\right)$ from the summation and the integration is less than $5 \times 10^{-4}$. With the lattice data that include the excited state contributions, we could expect a larger error, $\sim 1 \times 10^{-3}$, which is estimated from a difference between VMD and lattice data in Fig. 1. We can therefore safely neglect this source of error as it is well below $1 \%$.

We use two lattice volumes and two topological-charge sectors to check finite-size (FS) effects. Following Ref. [20], we analyze the fixed-topology (FT) effect and find it suppressed due to the kinematical structure of $\varepsilon_{\mu \nu \alpha \beta} p_{1}^{\alpha} p_{2}^{\beta}$. By comparing the lattice results at different topological-charge sectors, we do not observe statistically significant FT effects. The leading FS effect in $C_{\mu \nu}\left(t_{1}, t_{2}, t_{\pi}\right)$ is the conventional one and known to behave as $e^{-m_{\pi} L}$ [23]. To reduce the contamination due to this effect, we therefore use the data with $m_{\pi} L \geq 4$ to perform the chiral extrapolation. (Namely, we exclude the $L / a=$ 16 data points at the lowest two pion masses.)

$\chi \mathrm{PT}$ shows that up to next-to-leading order (NLO) the $m_{\pi}$ dependence of $F\left(m_{\pi}^{2}, 0,0\right)$ involves no chiral logarithm [24,25]. We therefore simply fit $F\left(m_{\pi}^{2}, 0,0\right)$ by a linear function in $m_{\pi}^{2}$, and obtain $F(0,0,0)=1.016(20)$ and $F\left(m_{\pi, \text { phy }}^{2}, 0,0\right)=1.011(19)$. To check the higher-order correction, we also perform a quadratic fit under the constraint from the ABJ anomaly: $F(0,0,0)=1$. We do not find any statistically significant difference due to the higher-order term. The linear (quadratic) fit is shown by the solid (dashed) line in the uppermost panel of Fig. 3. 
Next we consider the data with $m_{\pi} L<4$, which tend to suffer from the FS effect. As shown in Fig. 3, at $m_{\pi} \approx 290 \mathrm{MeV}$ we find that $F\left(m_{\pi}^{2}, 0,0\right)$ calculated at the $L / a=16$ lattice is $27 \%$ less than the one at $L / a=24$. Although large, such FS effect is understandable. By inserting the ground state into $\left\langle j_{\mu} j_{\nu} \pi^{0}\right\rangle$, we can approximate this three-point correlation function with three hadronic matrix elements,

$$
\left\langle j_{\mu} j_{\nu} \pi^{0}\right\rangle \rightarrow\left\langle\Omega\left|j_{\mu}\right| V, \varepsilon\right\rangle\left\langle V, \varepsilon\left|j_{\nu}\right| \pi^{0}\right\rangle\left\langle\pi^{0}\left|\pi^{0}\right| \Omega\right\rangle .
$$

The first matrix element is related to the electromagnetic coupling $g_{V}$ as $\left\langle\Omega\left|j_{\mu}\right| V, \varepsilon\right\rangle=M_{V}^{2} g_{V} \varepsilon_{\mu}$, the second is proportional to the $V \pi \gamma$ coupling $g_{V \pi \gamma}$, and the third is related to $F_{\pi}$ by the partially-conserved-axial-vectorcurrent constraint (PCAC) relation. In our calculation, we do not observe a significant FS effect in $M_{V}$ but find $8 \%$, $7 \%$, and $9 \%$ shifts in $g_{V}, g_{V \pi \gamma}$, and $F_{\pi}$, respectively, from $L / a=16$ to 24 , as shown in Fig. 3. These FS effects may accumulate in the three-point function. We estimate the FS corrections $R_{\mathcal{O}} \equiv \mathcal{O}(\infty) / \mathcal{O}(L)$ with $\mathcal{O}=g_{V}, g_{V \pi \gamma}$, and $F_{\pi} . R_{g_{V}}$ and $R_{g_{V \pi \gamma}}$ are evaluated by adding a correction term, $e^{-m_{\pi} L}$, into the linear fit form in the chiral extrapolation of each quantity. With such corrections taken into account, we confirm that their chiral limit is consistent with experimental data. $R_{F_{\pi}}$ is calculated to NNLO by using $\chi \mathrm{PT}$ [26]. Assuming that $R_{F\left(m_{\pi}^{2}, 0,0\right)}=R_{g_{V}} R_{g_{V \pi \gamma}} R_{F_{\pi}}$, we may correct $F\left(m_{\pi}^{2}, 0,0\right)$ by a factor of $R_{F\left(m_{\pi}^{2}, 0,0\right)}$. As shown in the lowest panel of Fig. 3, with FS correction $F\left(m_{\pi}^{2}, 0,0\right)$ at $L / a=16$ agrees with those at $L / a=24$. Using the corrected data to perform a linear extrapolation, we obtain $F(0,0,0)=1.045(35)$ and $F\left(m_{\pi, \text { phy }}^{2}, 0,0\right)=1.041(32)$. The difference between the results from the two methods is considered as a systematic error.

So far, our results are obtained neglecting the effect of disconnected diagrams that may appear because the electromagnetic current $j_{\mu}$ contains a flavor-singlet contribution. Calculation of the disconnected diagram is computationally demanding and statistically noisy. We solve these problems by the use of the all-to-all propagator. The full data, including both the connected and disconnected contributions, are plotted in the upper (lower) panel of Fig. 4 for the case without (with) the FS correction. We find that, although not significant, there is a shift from the connected data to the full ones. Since the accuracy of our calculation reaches a few-percent level, the disconnected effect is relevant. Using the full data, we repeat the analysis. The linear fit of $F\left(m_{\pi}^{2}, 0,0\right)$ with $m_{\pi} L \geq 4$ yields $F(0,0,0)=1.009(22)$ and $F\left(m_{\pi, \text { phy }}^{2}, 0,0\right)=1.005(20)$. The fit with FS corrected $F\left(m_{\pi}^{2}, 0,0\right)$ produces $F(0,0,0)=$ $1.007(36)$ and $F\left(m_{\pi, \text { phy }}^{2}, 0,0\right)=1.006(33)$. Including the disconnected contributions, the normalized form factor in the chiral limit and at the physical pion mass shifts by $1-4 \%$. This is comparable to the statistical error.

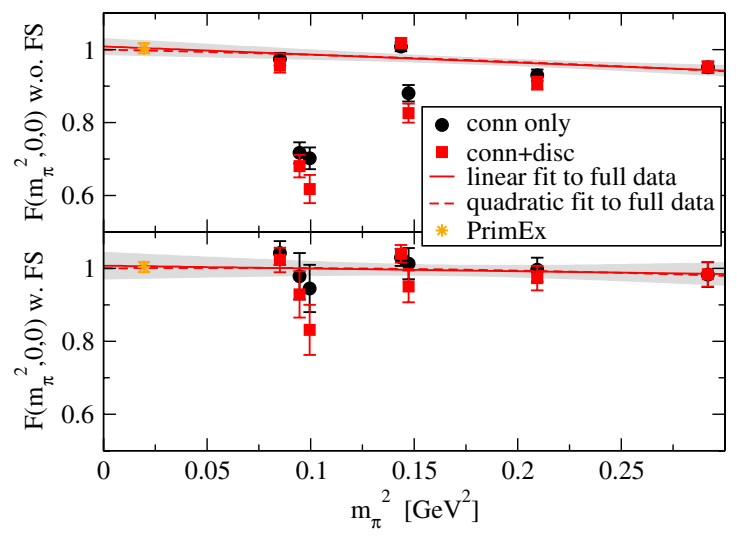

FIG. 4 (color online). A comparison of the full contribution to $F\left(m_{\pi}^{2}, 0,0\right)$ and the connected-only piece. The upper (lower) panel shows the result without (with) FS correction.

Using the full data, we quote our results for $F\left(m_{\pi}^{2}, 0,0\right)$ and $\Gamma_{\pi^{0} \gamma \gamma}$ in the isospin symmetric limit as

$$
\begin{aligned}
F(0,0,0) & =1.009(22)(29), \\
F\left(m_{\pi, \text { phy }}^{2}, 0,0\right) & =1.005(20)(30), \\
\Gamma_{\pi^{0} \gamma \gamma} & =7.83(31)(49) \mathrm{eV},
\end{aligned}
$$

where the systematic errors originate from the difference of the results by using two methods of treating the FS effect. (The difference appearing in the full data is small. To be conservative, we use the connected data to estimate such systematic error.) Our results reproduce the predication of the ABJ anomaly $F(0,0,0)=1$ and agree with the PrimEx Collaboration measurement $\Gamma_{\pi^{0} \gamma \gamma}=7.82(22) \mathrm{eV}$ [4]. For future improvements, isospin breaking effects due to the light quark mass difference need to be included.

Numerical simulations were performed on the Hitachi SR16000 at Yukawa Institute of Theoretical Physics and at High Energy Accelerator Research Organization under support of its Large Scale Simulation Program (No. 10-11). This work is supported in part by Grant-in-Aid of the Japanese Ministry of Education (Nos. 21674002 and 21684013), the Grant-in-Aid for Scientific Research on Innovative Areas (Nos. 2004: 20105001, 20105002, 20105003, 20105005, and 23105710]), and the HPCI Strategic Program of the Japanese Ministry of Education.

[1] S. L. Adler, Phys. Rev. 177, 2426 (1969).

[2] J. S. Bell and R. Jackiw, Nuovo Cimento A 60, 47 (1969).

[3] S. L. Adler and W. A. Bardeen, Phys. Rev. 182, 1517 (1969).

[4] I. Larin et al. (PrimEx Collaboration), Phys. Rev. Lett. 106, 162303 (2011).

[5] B. Ioffe and A. Oganesian, Phys. Lett. B 647, 389 (2007).

[6] J. L. Goity, A. M. Bernstein, and B. R. Holstein, Phys. Rev. D 66, 076014 (2002). 
[7] B. Ananthanarayan and B. Moussallam, J. High Energy Phys. 05 (2002) 052.

[8] K. Kampf and B. Moussallam, Phys. Rev. D 79, 076005 (2009).

[9] A. Bernstein and B. R. Holstein arXiv:1112.4809.

[10] H. Neuberger, Phys. Lett. B 417, 141 (1998).

[11] K. Fujikawa and M. Ishibashi, Nucl. Phys. B587, 419 (2000).

[12] S. D. Cohen, H. W. Lin, J. Dudek, and R. G. Edwards, Proc. Sci., LATTICE2008 (2008) 159.

[13] E. Shintani, S. Aoki, S. Hashimoto, T. Onogi, and N. Yamada, Proc. Sci., LATTICE2010 (2010) 159.

[14] J. Foley, K. J. Juge, A. Ó. Cais, M. Peardon, S. M. Ryan, and J.-I. Skullerud, Comput. Phys. Commun. 172, 145 (2005).

[15] T. Kaneko et al. (JLQCD Collaboration), Proc. Sci., LATTICE2010 (2010) 146.

[16] X.D. Ji and C.W. Jung, Phys. Rev. Lett. 86, 208 (2001).

[17] J. J. Dudek and R. G. Edwards, Phys. Rev. Lett. 97, 172001 (2006).
[18] H. Matsufuru et al. (JLQCD Collaboration and TWQCD Collaboration), Proc. Sci., LATTICE2008 (2008) 077.

[19] J. Noaki et al. (JLQCD Collaboration and TWQCD Collaboration, Proc. Sci. LATTICE2010 (2010) 117.

[20] S. Aoki, H. Fukaya, S. Hashimoto, and T. Onogi, Phys. Rev. D 76, 054508 (2007).

[21] J. Noaki, T. W. Chiu, H. Fukaya, S. Hashimoto, H. Matsufuru, T. Onogi, E. Shintani, and N. Yamada, Phys. Rev. D 81, 034502 (2010).

[22] X. Feng, S. Aoki, H. Fukaya, S. Hashimoto, T. Kaneko, J. Noaki, and E. Shintani (to be published).

[23] J. Gasser and H. Leutwyler, Nucl. Phys. B307, 763 (1988).

[24] J. F. Donoghue, B. R. Holstein, and Y. C. R. Lin, Phys. Rev. Lett. 55, 2766 (1985).

[25] J. Bijnens, A. Bramon, and F. Cornet, Phys. Rev. Lett. 61, 1453 (1988).

[26] G. Colangelo, S. Durr, and C. Haefeli, Nucl. Phys. B721, 136 (2005).

[27] K. Nakamura et al. (Particle Data Group), J. Phys. G 37, 075021 (2010). 